\title{
Second Order Periodic Boundary Value Problems Involving the Distributional Henstock-Kurzweil Integral*
}

\author{
Xueyuan Zhou, Guoju Ye \\ Department of Mathematics, Hohai University, Nanjing, China \\ Email: xyzhouhhu@163.com,yegj@hhu.edu.cn
}

Received May 25, 2012; revised June 25, 2012; accepted July 4, 2012

\begin{abstract}
We apply the distributional derivative to study the existence of solutions of the second order periodic boundary value problems involving the distributional Henstock-Kurzweil integral. The distributional Henstock-Kurzweil integral is a general intergral, which contains the Lebesgue and Henstock-Kurzweil integrals. And the distributional derivative includes ordinary derivatives and approximate derivatives. By using the method of upper and lower solutions and a fixed point theorem, we achieve some results which are the generalizations of some previous results in the literatures.
\end{abstract}

Keywords: Periodic Boundary Value Problem; Distributional Henstock-Kurzweil Integral; Distributional Derivative; Existence; Upper and Lower Solutions; Fixed Point

\section{Introduction}

This paper is devoted to the study of the existence of solutions of the second order periodic boundary value problem (PBVP for brevity)

$$
\begin{aligned}
& -D^{2} x=f(t)+g(t, x, D x), \\
& x(0)=x(T), D x(0)=D x(T)=0,
\end{aligned}
$$

where $D x$ and $D^{2} x$ are the first and second order distributional derivatives of $x \in C^{1}([0, T])$ respectively, $g:[0, T] \times C^{1}([0, T]) \times C([0, T]) \rightarrow \mathbb{R}$ and $f$ is a distribution (generalized function).

If the distributional derivative in the system (1.1) is replaced by the ordinary derivative and $f(t)=0$, then (1) converts into

$$
\begin{aligned}
& -x^{\prime \prime}=g\left(t, x, x^{\prime}\right), \\
& x(0)=x(T), \quad x^{\prime}(0)=x^{\prime}(T),
\end{aligned}
$$

here $g:[0, T] \times \mathbb{R} \times \mathbb{R} \rightarrow \mathbb{R}$, and $x^{\prime}$ and $x^{\prime \prime}$ denote the first and second ordinary derivatives of $x \in C^{2}([0, T])$. The existence of solutions of (1.2) have been extensively studied by many authors $[1,2]$. It is well-known, the notion of a distributional derivative is a general concept, including ordinary derivatives and approximate derivatives. As far as we know, few papers have applied distributional derivatives to study PBVP. In this paper, we have come up with a new way, instead of the ordinary derivative, using the distributional derivative to study the

* Supported by NNSF of China (10871059) and the Fundamental Research Funds for the Central Universities.
PBVP and obtain some results of the existence of solutions.

This paper is organized as follows. In Section 2, we introduce fundamental concepts and basic results of the distributional Henstock-Kurzweil integral or briefly the $D_{H K}$-integral. A distribution $f$ is $D_{H K}$-integrable on $[a, b] \subset \mathbb{R}$ if there is a continuous function $F$ on $[a, b]$ with $F(a)=0$ whose distributional derivative equals $f$. From the definition of the $D_{H K}$-integral, it includes the Riemann integral, Lebesgue integral, $H K$-integral and wide Denjoy integral (for details, see [3-5]). Furthermore, the space of $D_{H K}$-integrable distributions is a Banach space and has many good properties, see [6-8].

In Section 3, with the $D_{H K}$-integral and the distributional derivative, we generalize the PBVP (1.2) to (1.1). By using the method of upper and lower solutions and a fixed point theorem, we achieve some interesting results which are the generalizations of some corresponding results in the references.

\section{The Distributional Henstock-Kurzweil Integral}

In this section, we present the definition and some basic properties of the distributional Henstock-Kurzweil integral.

Define the space

$C_{c}^{\infty}=$

$\left\{\phi: \mathbb{R} \rightarrow \mathbb{R} \mid \phi \in C^{\infty}\right.$ and $\phi$ has compact support in $\left.\mathbb{R}\right\}$, where the support of a function $\phi$ is the closure of the 
set on which $\phi$ does not vanish, denote by $\operatorname{supp}(\phi)$. A sequence $\left\{\phi_{n}\right\} \subset C_{c}^{\infty}$ converges to $\phi \in C_{c}^{\infty}$ if there is a compact set $K$ such that all $\phi_{n}$ have support in $K$ and for every $m \in \mathbb{N}$ the sequence of derivatives $\phi_{n}^{(m)}$ converges to $\phi^{(m)}$ uniformly on $K$. Denote $C_{c}^{\infty}$ endowed with this convergence property by $\mathcal{D}$. Where $\phi$ is called test function if $\phi \in \mathcal{D}$. The distributions are defined as continuous linear functionals on $\mathcal{D}$. The space of distributions is denoted by $\mathcal{D}^{\prime}$, which is the dual space of $\mathcal{D}$. That is, if $f \in \mathcal{D}^{\prime}$ then $f: \mathcal{D} \rightarrow \mathbb{R}$, and we write $\langle f, \phi\rangle \in \mathbb{R}$, for $\phi \in \mathcal{D}$.

For all $f \in \mathcal{D}^{\prime}$, we define the distributional derivative Df of $f$ to be a distribution satisfying

$\langle D f, \phi\rangle=-\left\langle f, \phi^{\prime}\right\rangle$, where $\phi$ is a test function.

Let $(a, b)$ be an open interval in $\mathbb{R}$, we define

$$
\begin{aligned}
\mathcal{D}((a, b)) & =\left\{\phi:(a, b) \rightarrow \mathbb{R} \mid \phi \in C^{\infty}\right. \\
& \text { and } \phi \text { has compact support in }(a, b)\},
\end{aligned}
$$

the dual space of $\mathcal{D}((a, b))$ is denoted by $\mathcal{D}^{\prime}((a, b))$.

Remark 2.1. $\mathcal{D}((a, b))$ and $\mathcal{D}^{\prime}((a, b))$ are $\mathcal{D}$ and $\mathcal{D}^{\prime}$ respectively if $a=-\infty, b=+\infty$.

Let $C([a, b])$ be the space of continuous functions on $[a, b]$, and

$$
B_{C}=\{F \in C([a, b]): F(a)=0\} .
$$

Note that $B_{C}$ is a Banach space with the uniform norm $\|F\|_{\infty}=\max _{[a, b]}|F|$.

Now we are able to introduce the definition of the $D_{H K}$-integral.

Definition 2.1. A distribution $f$ is distributionally Henstock-Kurzweil integrable or briefly $D_{H K}$-integrable on $[a, b]$ if $f$ is the distributional derivative of $a$ continuous function $F \in B_{C}$.

The $D_{H K}$-integral of $f$ on $[a, b]$ is denoted by $\left(D_{H K}\right) \int_{a}^{b} f=F(b)$, where $F$ is called the primitive of $f$ and " $\left(D_{H K}\right) \int$ " denotes the $D_{H K}$-integral. Analogously, we denote $H K$-integral and Lebesgue integral.

The space of $D_{H K}$-integrable distributions is defined by

$$
D_{H K}=\left\{f \in \mathcal{D}^{\prime}((a, b)): f=D F, F \in B_{C}\right\} .
$$

With this definition, if $f \in D_{H K}$ then we have for all $\phi \in \mathcal{D}((a, b))$.

$$
\langle f, \phi\rangle=\langle D F, \phi\rangle=-\left\langle F, \phi^{\prime}\right\rangle=-\int_{a}^{b} F \phi^{\prime} .
$$

With the definition above, we know that the concept of the $D_{H K}$-integral leads to its good properties. We firstly mention the relation between the $D_{H K}$-integral and the $H K$-integral.

Recall that $f$ is Henstock-Kurzweil integrable on $[a, b]$ if and only if there exists a continuous function $F$ which is $A C G^{*}$ (generalized absolutely continuous, see [4]) on $[a, b]$ such that $F^{\prime}(x)=f(x)$ almost everywhere. P. Y. Lee pointed out that if $F$ is a continuous function and pointwise differentiable nearly everywhere on $[a, b]$, then $F$ is $A C G^{*}$. Furthermore, if $F$ is a continuous function which is differentiable nowhere on $[a, b]$, then $F$ is not $A C G^{*}$. Therefore, if $F \in C([a, b])$ but differentiable nowhere on $[a, b]$, then $D F$ exists and is $D_{H K}$-integrable but not $H K$ integrable. Conversely, if $F \in A C G^{*}$ and it also belongs to $C([a, b])$. Then $F^{\prime}$ is not only $H K$-integrable but also $D_{H K}$-integrable. Here $F^{\prime}$ denotes the ordinary derivative of $F$. Obviously, the $D_{H K}$-integral includes the $H K$-integral.

Now we shall give some corresponding results of the distributional Henstock-Kurzweil integral.

Lemma 2.1. ([3, Theorem 4], Fundamental Theorem of Calculus).

1) Let $f \in D_{H K}$, define $F(t)=\left(D_{H K}\right) \int_{a}^{t} f$. Then $F \in B_{C}$ and $D F=f$.

2) Let $F \in C([a, b])$. Then $\left(D_{H K}\right) \int_{a}^{t} D F=F(t)-F(a)$ for all $t \in[a, b]$.

For $f \in D_{H K}$, we define the Alexiewicz norm by

$$
\|f\|=\|F\|_{\infty}=\max _{[a, b]}|F| \text {. }
$$

The following result has been proved.

Lemma 2.2. ([3, Thoerem 2]). With the Alexiewicz norm, $D_{H K}$ is a Banach space.

We now impose a partial ordering on $D_{H K}$ : for $f, g \in D_{H K}$, we say that $f \succeq g \quad$ (or $g \preceq f$ ) if and only if $f-g$ is a measure on $[a, b]$ (see details in [9]). By this definition, if $f, g \in D_{H K}$ then

$$
\left(D_{H K}\right) \int_{I} f \geq\left(D_{H K}\right) \int_{I} g,
$$

whenever $f \succeq g, I=[c, d] \subset[a, b]$. We also have other usual relations between the $D_{H K}$-integral and the ordering, for instance, the following result.

Lemma 2.3. ([9, Corollary 1]). If $f, g, h \in \mathcal{D}^{\prime}((a, b))$, $f \preceq g \preceq h$ and if $f$ and $h$ are $D_{H K}$-integrable, then $g$ is also $D_{H K}$-integrable.

We say a sequence $\left\{f_{n}\right\} \subset D_{H K}$ converges strongly to $f \in D_{H K}$ if $\left\|f_{n}-f\right\| \rightarrow 0$ as $n \rightarrow \infty$. It is also shown that the following two convergence theorems hold.

Lemma 2.4. ([9, Corollary 4], Monotone convergence theorem for the $D_{H K}$-integral). Let $\left\{f_{n}\right\}_{n=0}^{\infty}$ be a sequence in $D_{H K}$ such that $f_{0} \preceq f_{1} \preceq \cdots \preceq f_{n} \preceq \cdots$ and that $\left(D_{H K}\right) \int_{a}^{b} f_{n} \rightarrow A$ as $n \rightarrow \infty$. Then $f_{n} \rightarrow f$ in $D_{H K}$ and $\left(D_{H K}\right) \int_{a}^{b} f=A$.

Lemma 2.5. ([7, Lemma 2.3], Dominated convergence theorem for the $D_{H K}$-integral). Let $\left\{f_{n}\right\}_{n=0}^{\infty}$ be a sequence in $D_{H K}$ such that $f_{n} \rightarrow f$ in $\mathcal{D}^{\prime}$. Suppose there exist $g, h \in D_{H K}$ satisfying $g \preceq f \preceq h, \forall n \in \mathbb{N}$. 
Then $f \in D_{H K}$ and $\lim _{n \rightarrow \infty}\left(D_{H K}\right) \int_{a}^{b} f_{n}=\left(D_{H K}\right) \int_{a}^{b} f$.

We now give another result about the distributional derivative.

Lemma 2.6. Let $f, g$ be the distributional derivative of $F, G$, where $F, G \in C([a, b])$. Then

$$
D(F G)=f G+F g \text {. }
$$

Proof. It follows from the definition of the distributional derivative and (3.1) that

$$
\begin{aligned}
& \langle D(F G), \phi\rangle \\
& =-\left\langle F G, \phi^{\prime}\right\rangle=-\int_{a}^{b} F\left(G \phi^{\prime}\right)=-\int_{a}^{b} F(D(G \phi)-g \phi) \\
& =-\int_{a}^{b} F D(G \phi)+\int_{a}^{b} F g \phi=\int_{a}^{b} f G \phi+\int_{a}^{b} F g \phi \\
& =\int_{a}^{b}(F g+f G) \phi=\langle F g+f G, \phi\rangle .
\end{aligned}
$$

Consequently, the result holds. $\square$

If $g:[a, b] \rightarrow \mathbb{R}$, its variation is

$V g=\sup \sum_{n}\left|g\left(t_{n}\right)-g\left(s_{n}\right)\right|<+\infty$ where the supremum is taken over every sequence $\left\{\left(t_{n}, s_{n}\right)\right\}$ of disjoint intervals in $[a, b]$, then $g$ is called a function with bounded variation. The set of functions with bounded variation is denoted $\mathcal{B V}$. It is known that the dual space of $D_{H K}$ is $\mathcal{B V}$ (see details in [3]), and the following statement holds.

Lemma 2.7. ([3, Definition 6], Integration by parts). Let $f \in D_{H K}$, and $g \in \mathcal{B} \mathcal{V}$. Define $f g=D H$, where $H(t)=F(t) g(t)-\int_{a}^{t} F d g$. Then $f g \in D_{H K}$ and

$$
\int_{a}^{b} f g=F(b) g(b)-\int_{a}^{b} F d g .
$$

\section{Periodic Boundary Value Problems}

Consider the second order periodic boundary value problem (1.1)

$$
\begin{aligned}
& -D^{2} x=f(t)+g(t, x, D x), \\
& x(0)=x(T), \quad D x(0)=D x(T)=0,
\end{aligned}
$$

where $D x$ and $D^{2} x$ denote the first and second order distributional derivatives of $x \in C^{1}([0, T])$, respectively, $g:[0, T] \times C^{1}([0, T]) \times C([0, T]) \rightarrow \mathbb{R}$ and $f$ is a distribution (generalized function).

The distributional derivative subsumes the ordinary derivative. And if the first ordinary derivative of $x \in C^{1}([0, T])$ exists, the first ordinary derivative and first order distributional derivative of $x \in C^{1}([0, T])$ are equivalent. For $x \in C^{1}([0, T])$, then the distributional derivative $D x \in C([0, T])$ and $D x(0)=0$, hence $D^{2} x \in D_{H K}$.

Recall that we say $(v, D v) \leq(u, D u)$ if and only if $v(t) \leq u(t)$ and $D v(t) \leq D u(t)$ for all $t \in[0, T]$.
We impose the following hypotheses on the functions $f$ and $g$.

(D0) There exist $v, u \in C^{1}([0, T])$ with $(v, D v) \leq(u, D u), c_{v}, c_{u} \in D_{H K}$ such that

$$
\begin{aligned}
& -D^{2} u \preceq f+g(\cdot, u, D u)-c_{u}, \\
& -D^{2} v \succeq f+g(\cdot, v, D v)+c_{v}, \\
& u(T) \leq u(0), v(T) \geq v(0), \text { on }[0, T]
\end{aligned}
$$

and $p(t) \in H K, \quad p(t) \geq 0$, with $P(t)=(H K) \int_{0}^{t} p(s) \mathrm{d} s$ and $P(T) \neq 0, t \in[0, T]$ such that

$$
\begin{aligned}
& \operatorname{Du}(T)-D u(0) \\
& \leq\left(D_{H K}\right) \int_{t}^{T} e^{P(s)-P(T)} c_{u}(s) \mathrm{d} s+\left(D_{H K}\right) \int_{0}^{t} e^{P(s)} c_{u} \mathrm{~d} s, \\
& D v(0)-D v(T) \\
& \leq\left(D_{H K}\right) \int_{t}^{T} e^{P(s)-P(T)} c_{v}(s) \mathrm{d} s+\left(D_{H K}\right) \int_{0}^{t} e^{P(s)} c_{v}(s) \mathrm{d} s,
\end{aligned}
$$

(D1) $g(\cdot, x(\cdot), y(\cdot))$ is Lesbesgue integrable on $[0, T]$ when $x, y \in C^{1}([0, T]), v \leq x \leq u, D v \leq y \leq D u$, and $f$ is $D_{H K}$-integrable on $[0, T]$,

(D2) $g(t, x, y)-p(t) y$ is nonincreasing with respect to $(x, y) \in[v(t), u(t)] \times[D v(t), D u(t)]$ for all $t \in[0, T]$.

We say that $x$ is a solution of PBVP (1) if $x \in C^{1}([0, T])$ and satisfies (1). Before giving our main results in this paper, we first apply Lemma 2.1 to convert the PBVP (1) into an integral equation.

Lemma 3.1. Let $f:[0, T] \rightarrow \mathbb{R}$ be a distribution and $g:[0, T] \times C^{1}([0, T]) \times C([0, T]) \rightarrow \mathbb{R}$, a function $x:[0, T] \rightarrow \mathbb{R}$ is a solution of the PBVP (1.1) on $[0, T]$ if and only if $x$ and $D x=y$ satisfy for any $p \in H K$, $p(t) \geq 0$ on $[0, T]$, with $P(t)=(H K) \int_{0}^{t} p(s) \mathrm{d} s$ and $P(T) \neq 0$, the integral equation

$$
(x, y)=G(x, y)=\left(G_{1}(x, y), G_{2}(x, y)\right),
$$

where

$$
\begin{aligned}
& G_{1}(x, y)(t)=e^{-P(t)}\left(D_{H K}\right) \int_{0}^{t} e^{P(s)}(p(s) x(s)+y(s)) \mathrm{d} s \\
& +\frac{e^{-P(t)}}{e^{P(T)}-1}\left(D_{H K}\right) \int_{0}^{T} e^{P(s)}(p(s) x(s)+y(s)) \mathrm{d} s,
\end{aligned}
$$

and

$$
\begin{aligned}
G_{2}(x, y)(t)= & e^{-P(t)}\left(D_{H K}\right) \int_{0}^{t} e^{P(s)}(p(s) y(s)-f(s) \\
& -g(s, x(s), y(s))) d s \\
& +\frac{e^{-P(t)}}{e^{P(T)}-1}\left(D_{H K}\right) \int_{0}^{T} e^{P(s)} p(s) y(s) \\
& -f(s)-g(s, x(s), y(s))) \mathrm{d} s .
\end{aligned}
$$


Proof. Let $x \in C^{1}([0, T])$, then the function $y=D x$ with $D x(0)=0$ is continuous on $[0, T]$, so $D^{2} x$ is $D_{H K}$-integrable. Let $D x=y$, then by (1.1) we have $D y=-f(t)-g(t, x, y)$, or equivalently,

$$
\begin{aligned}
& e^{P(t)}(D y+p y) \\
& =e^{P(t)}(p y-f(t)-g(t, x, y))
\end{aligned}
$$

Integrating (3.4) we have

$$
\begin{aligned}
& e^{P(t)} y(t) \\
&= y(0)+\left(D_{H K}\right) \int_{0}^{t} e^{P(s)}(p(s) y(s) \\
&-f(s)-g(s, x(s), y(s))) \mathrm{d} s, \\
& y(0)= y(T) \\
&=\left(e^{P(T)}-1\right)^{-1}\left(D_{H K}\right) \\
& \int_{0}^{T} e^{P(s)}(p(s) y(s)-f(s)-g(s, x(s), y(s))) \mathrm{d} s .
\end{aligned}
$$

This implies $y=G_{2}(x, y)$. We can prove that $x=G_{1}(x, y)$ by the same way. Thus $x$ and $y=D x$ satisfy the operator Equation (3.1).

Conversely, assume that $x, y$ satisfy (3.1). In view of (2) we then have for each $t \in[0, T]$

$$
\begin{aligned}
& e^{P(t)} x(t) \\
& =\frac{1}{e^{P(T)}-1}\left(D_{H K}\right) \int_{0}^{T} e^{P(s)}((p(s) x(s)+y(s)) \mathrm{d} s \\
& \quad+\left(D_{H K}\right) \int_{0}^{t} e^{P(s)}(p(s) x(s)+y(s)) \mathrm{d} s .
\end{aligned}
$$

Noticing that $x, y \in C[0, T]$, then (3.5) implies by differentiation that

$$
D x=y \quad \text { on } \quad[0, T] .
$$

It follows from (3.1) and (3.3) that for each $t \in[0, T]$,

$$
\begin{aligned}
e^{P(t)} y(t) & \left(D_{H K}\right) \int_{0}^{t} e^{P(s)}(p(s) y(s)-f(s)-g(s, x(s), y(s))) \mathrm{d} s \\
& +\frac{1}{e^{P(T)}-1}\left(D_{H K}\right) \\
& \int_{0}^{T} e^{P(s)}(p(s) y(s)-f(s)-g(s, x(s), y(s))) \mathrm{d} s .
\end{aligned}
$$

Applying Lemma 2.6 to (3.7), we obtain for all $t \in[0, T]$

$$
\begin{aligned}
e^{P(t)} G_{1} \psi(t) & =\left(e^{P(T)}-1\right)^{-1}\left(D_{H K}\right) \int_{0}^{T} e^{P(s)}(p(s) u(s)+D u(s)) \mathrm{d} s+\left(D_{H K}\right) \int_{0}^{t} e^{P(s)}(p(s) u(s)+D u(s)) \mathrm{d} s \\
& =\left(e^{P(T)}-1\right)^{-1}\left(e^{P(T)} u(T)-u(0)\right)+e^{P(t)} u(t)-u(0) \leq u(0)+e^{P(t)} u(t)-u(0)=e^{P(t)} u(t) .
\end{aligned}
$$

It follows from (3.7), (3.10) and (D0) that for each $t \in[0, T]$

$$
\begin{aligned}
& e^{P(t)}(p(t) y(t)+D y(t)) \\
& =e^{P(t)}(p(t) y(t)-f(s)-g(t, x(t), y(t))),
\end{aligned}
$$

which together with (3.6) implies that

$$
D^{2} x=-f(t)-g(t, x, D x), \quad t \in[0, T] .
$$

It follows from (5) that $x(T)=x(0)$, and from (7) that $D x(T)=D x(0)$, so that $x$ is a solution of the PBVP (1.1).

Let $E$ be an ordered Banach space, $K$ a nonempty subset of $E$. The mapping $G: K \rightarrow E$ is increasing if and only if $G \varphi \leq G \psi$, whenever $\varphi, \psi \in K$ and $\varphi \leq \psi$.

An important tool which will be used latter concerns a fixed point theorem for an increasing mapping and is stated next.

Lemma 3.2. ([10, Theorem 3.1.3]) Let $\varphi_{0}, \psi_{0} \in E$ with $\varphi_{0}<\psi_{0}$, and $G:\left[\varphi_{0}, \psi_{0}\right] \rightarrow E$ be an increasing mapping satisfying $\varphi_{0} \leq G \varphi_{0}, G \psi_{0} \leq \psi_{0}$. If

$G\left(\left[\varphi_{0}, \psi_{0}\right]\right)$ is relatively compact, then $G$ has a maximal fixed point $x^{*}$ and a minimal fixed point $x_{*}$ in $\left[\varphi_{0}, \psi_{0}\right]$. Moreover,

$$
X_{*}=\lim _{n \rightarrow \infty} \varphi_{n}, x^{*}=\lim _{n \rightarrow \infty} \psi_{n},
$$

where $\varphi_{n}=G \varphi_{n-1}$ and $\psi_{n}=G \psi_{n-1}(n=1,2,3, \cdots)$,

$$
\begin{aligned}
& \varphi_{0} \leq \varphi_{1} \leq \cdots \leq \varphi_{n} \leq \cdots \leq \\
& x_{*} \leq x^{*} \leq \cdots \leq \psi_{n} \leq \cdots \leq \psi_{1} \leq \psi_{0} .
\end{aligned}
$$

Lemma 3.3. Let conditions (D0)-(D2) be satisfied. Denoting

$$
\begin{aligned}
& \varphi(t)=(v(t), D v(t)), \\
& \psi(t)=(u(t), D u(t)), t \in[0, T],
\end{aligned}
$$

then $G \psi \leq \psi$ and $\varphi \leq G \varphi$.

Proof. The hypotheses $(D 0)$ and $(D 2)$ imply that for all $x, y$ in $C([0, T])$, satisfying

$$
\begin{gathered}
(v, D v) \leq(x, y) \leq(u, D u), \\
D^{2} v+c_{v}+p D v \preceq p y-f(t) \\
-g(t, x, y) \preceq D^{2} u-c_{u}+p D u, \quad t \in[0, T] .
\end{gathered}
$$

This and (D1) ensure that $G_{j} \varphi$ and $G_{j} \psi$ in (3.2) and (3.3) are defined for $j=1,2$. Condition (D0) implies that for each $t \in[0, T]$ 


$$
\begin{aligned}
e^{P(t)} G_{2} \psi(t)= & \left(D_{H K}\right) \int_{0}^{t} e^{P(s)}(p(s) D u(s)-f(s)-g(s, u(s), D u(s))) \mathrm{d} s \\
+ & \frac{1}{e^{P(T)}-1}\left(D_{H K}\right) \int_{0}^{T} e^{P(s)}(p(s) D u(s)-f(s)-g(s, u(s), D u(s))) \mathrm{d} s \\
\leq & \left(D_{H K}\right) \int_{0}^{t} e^{P(s)}\left(p(s) D u(s)+D^{2} u(s)-c_{u}(s)\right) \mathrm{d} s \\
& +\frac{1}{e^{P(T)}-1}\left(D_{H K}\right) \int_{0}^{T} e^{P(s)}\left(p(s) D u(s)+D^{2} u(s)-c_{u}(s)\right) \mathrm{d} s \\
= & e^{P(t)} D u(t)-D u(0)-\left(D_{H K}\right) \int_{0}^{t} e^{P(s)} c_{u}(s) \mathrm{d} s+\frac{1}{e^{P(T)}-1}\left(e^{P(T)} D u(T)-D u(0)-\left(D_{H K}\right) \int_{0}^{T} e^{P(s)} c_{u}(s) \mathrm{d} s\right) \\
= & e^{P(t)} D u(t)+\frac{e^{P(T)}}{e^{P(T)}-1}(D u(T)-D u(0))-\left(e^{P(T)}-1\right)^{-1}\left(D_{H K}\right) \int_{0}^{T} e^{P(s)} c_{u}(s) \mathrm{d} s \\
& -\left(D_{H K}\right) \int_{0}^{t} e^{P(s)} c_{u}(s) \mathrm{d} s \leq e^{P(t)} D u(t) .
\end{aligned}
$$

Thus, $G_{1} \psi \leq u$ and $G_{2} \psi \leq D u$, whence $G \psi \leq \psi$. The proof that $\varphi \leq G \varphi$ is similar. $\square$

Lemma 3.4. Assume that conditions (D0)-(D2) hold. Denoting

$$
\begin{aligned}
{[\phi, \psi] } & \left\{(x, y) \in C^{1}([0, T]) \times C([0, T]): \varphi \leq(x, y) \leq \psi\right\}, \quad \begin{array}{l}
\text { be given. The hypotheses }(D 0)-(D 2) \text { imply that for each } \\
t \in[0, T]
\end{array} \\
& e^{P(t)} G_{1}\left(x_{1}, y_{1}\right)(t)=\left(D_{H K}\right) \int_{0}^{t} e^{P(s)}\left(p(s) x_{1}(s)+y_{1}(s)\right) \mathrm{d} s+\frac{1}{e^{P(T)}-1}\left(D_{H K}\right) \int_{0}^{T} e^{P(s)}\left(p(s) x_{1}(s)+y_{1}(s)\right) \mathrm{d} s \\
& \leq\left(D_{H K}\right) \int_{0}^{t} e^{P(s)}\left(p(s) x_{2}(s)+y_{2}(s)\right) \mathrm{d} s+\frac{1}{e^{P(T)}-1}\left(D_{H K}\right) \int_{0}^{T} e^{P(s)}\left(p(s) x_{2}(s)+y_{2}(s)\right) \mathrm{d} s=e^{P(t)} G_{1}\left(x_{2}, y_{2}\right)(t),
\end{aligned}
$$

then the Equations (1)-(3) define a nondecreasing mapping $G:[\varphi, \psi] \rightarrow[\varphi, \psi]$.

Proof. Let

$$
\left(x_{1}, y_{1}\right),\left(x_{2}, y_{2}\right) \in[\varphi, \psi],\left(x_{1}, y_{1}\right) \leq\left(x_{2}, y_{2}\right),
$$

and

$$
\begin{aligned}
e^{P(t)} G_{2}\left(x_{1}, y_{1}\right)(t) \\
=\left(D_{H K}\right) \int_{0}^{t} e^{P(s)}\left(p(s) y(s)-f(s)-g\left(s, x_{1}(s), y_{1}(s)\right)\right) \mathrm{d} s \\
\quad+\frac{1}{e^{P(T)}-1}\left(D_{H K}\right) \int_{0}^{T} e^{P(s)}\left(p(s) y(s)-f(s)-g\left(s, x_{1}(s), y_{1}(s)\right) \mathrm{d} s\right. \\
\leq\left(D_{H K}\right) \int_{0}^{t} e^{P(s)}\left(p(s) y_{2}(s)-f(s)-g\left(s, x_{2}(s), y_{2}(s)\right) \mathrm{d} s\right. \\
\quad+\frac{1}{e^{P(T)}-1}\left(D_{H K}\right) \int_{0}^{T} e^{P(s)}\left(p(s) y_{2}(s)-f(s)-g\left(s, x_{2}(s), y_{2}(s)\right)\right) \mathrm{d} s=e^{P(t)} G_{2}\left(x_{2}, y_{2}\right)(t)
\end{aligned}
$$

Thus $G_{j}\left(x_{1}, y_{1}\right) \leq G_{j}\left(x_{2}, y_{2}\right), j=1,2$. This and Lemma 3.3 imply the assertion. $\square$

With the preparation above, we will prove our main result on the existence of the extremal solutions of the periodic boundary value problem (1.1).

Theorem 3.1. Assume that conditions $(D 0)-(D 2)$ are satisfied. Then the PBVP (1.1) has such solutions $\underline{x}$ and $\bar{x}$ in $[v, u]$ that $\underline{x} \leq x \leq \bar{x}$ and $D \underline{x} \leq D x \leq D \bar{x}$ for each solution $x$ of $(1.1)$ in $[v, u]$ such that $D x \in[D v, D u]$.

Proof. In view of Lemma 3.4 the Equations (3.1)-(3.3) define a nondecreasing mapping $G:[\varphi, \psi] \rightarrow[\varphi, \psi]$.
For any $(x, y) \in[\varphi, \psi]$, we have

$$
v \leq G_{1}(x, y) \leq u, D v \leq G_{2}(x, y) \leq D u, \text { on }[0, T] .
$$

Since $u, v \in C^{1}([0, T])$ and $D u, D v \in C([0, T])$, there exists constant $N_{1}$ such that, for each $(x, y) \in[\varphi, \psi]$,

$$
\begin{aligned}
& \left\|G_{1}(x, y)\right\| \leq\|v\|+\|u\| \leq N_{1}, \\
& \left\|G_{2}(x, y)\right\| \leq\|D v\|+\|D u\| \leq N_{1},
\end{aligned}
$$

which implies $G([\varphi, \psi])$ is uniformly bounded on $[0, T]$.

Let $t_{1}, t_{2} \in[0, T]$. Then by (3.2) and (3.3), for each $(x, y) \in[\varphi, \psi]$ 


$$
\begin{aligned}
& G_{1}(x, y)\left(t_{1}\right)-G_{1}(x, y)\left(t_{2}\right) \\
= & \left(e^{P\left(t_{2}\right)-P\left(t_{1}\right)}-1\right) G_{1}(x, y)\left(t_{2}\right) \\
& \quad+e^{-P\left(t_{1}\right)}\left(D_{H K}\right) \int_{t_{2}}^{t_{1}} e^{P(s)}(p(s) x(s)+y(s)) \mathrm{d} s \\
G_{2} & (x, y)\left(t_{1}\right)-G_{2}(x, y)\left(t_{2}\right) \\
= & \left(e^{P\left(t_{2}\right)-P\left(t_{1}\right)}-1\right) G_{2}(x, y)\left(t_{2}\right)+e^{-P\left(t_{1}\right)}\left(D_{H K}\right) \\
& \int_{t_{2}}^{t_{1}} e^{P(s)}(p(s) y(s)-f(s)-g(s, x(s), y(s))) \mathrm{d} s .
\end{aligned}
$$

Since $p(t) \in H K, \quad p(t) \geq 0$,

$P(t)=(H K) \int_{0}^{t} p(s) \mathrm{d} s$ is continuous and so is uniformly continuous on $[0, T]$, i.e., for all $\varepsilon>0$, there exists $\delta>0$ such that

$$
\begin{aligned}
& \left|P\left(t_{2}\right)-P\left(t_{1}\right)\right| \leq \varepsilon \\
& \text { whenever } t_{1}, t_{2} \in[0, T] \text { and }\left|t_{2}-t_{1}\right| \leq \delta .
\end{aligned}
$$

It is easy to see that $e^{P(t)} \in C([0, T]) \cap \mathcal{B V}$ (so is $e^{-P(t)}$ ) on $[0, T]$. Hence, there exists $M>0$ such that

$$
\frac{1}{M}<e^{P(t)}<M, t \in[0, T]
$$

The result $e^{P(t)} \in \mathcal{B V}$ on $[0, T]$ implies by Lemma 2.6 that $e^{P(t)}(p(t) x(t)+y(t))$ and $e^{P(t)}(p(t) y(t)-f(s)-g(t, x(t), y(t)))$ are

$D_{H K}$-integrable on $[0, T]$, because $p(t) x(t)+y(t)$ and $p(t) y(t)-f(t)-g(t, x(t), y(t))$ are $D_{H K}$-integrable for all $(x, y) \in[\varphi, \psi]$. This result and the monotonicity of $e^{P(t)}(p(t) x(t)+y(t))$ and $e^{P(t)}(p(t) y(t)-f(t)-g(t, x(t), y(t)))$ imply

$$
\left(D_{H K}\right) \int_{t_{2}}^{t_{1}} e^{P(s)}(p(s) v(s)+D v(s)) \mathrm{d} s \leq\left(D_{H K}\right) \int_{t_{2}}^{t_{1}} e^{P(s)}(p(s) x(s)+y(s)) \mathrm{d} s \leq\left(D_{H K}\right) \int_{t_{2}}^{t_{1}} e^{P(s)}(p(s) u(s)+D u(s)) \mathrm{d} s,
$$

and

$$
\begin{aligned}
& \left(D_{H K}\right) \int_{t_{2}}^{t_{1}} e^{P(s)}(p(s) D v(s)-f(s)-g(s, v(s), D v(s))) \mathrm{d} s \leq\left(D_{H K}\right) \int_{t_{2}}^{t_{1}} e^{P(s)}(p(s) y(s)-f(s)-g(s, x(s), y(s))) \mathrm{d} s \\
& \leq\left(D_{H K}\right) \int_{t_{2}}^{t_{1}} e^{P(s)}(p(s) D u(s)-f(s)-g(s, u(s), D u(s))) \mathrm{d} s .
\end{aligned}
$$

Then by (3.12)-(3.14), there exists $N_{2}>0$ such that

$$
\begin{aligned}
& \left|G_{1}(x, y)\left(t_{1}\right)-G_{1}(x, y)\left(t_{2}\right)\right| \leq M\left|\left(D_{H K}\right) \int_{t_{2}}^{t_{1}} e^{P(s)}(p(s) x(s)+y(s)) \mathrm{d} s\right|+N_{2} \varepsilon \\
& \leq M\left(\left|\left(D_{H K}\right) \int_{t_{2}}^{t_{1}} e^{P(s)}(p(s) v(s)+D v(s)) \mathrm{d} s\right|+\left|\left(D_{H K}\right) \int_{t_{2}}^{t_{1}} e^{P(s)}(p(s) u(s)+D u(s)) \mathrm{d} s\right|\right)+N_{2} \varepsilon,
\end{aligned}
$$

and

$$
\begin{aligned}
& \left|G_{2}(x, y)\left(t_{1}\right)-G_{2}(x, y)\left(t_{2}\right)\right| \leq M \mid\left(D_{H K}\right) \int_{t_{2}}^{t_{1}} e^{P(s)}\left(p(s) y(s)-f(s)-g(s, x(s), y(s)) d s \mid+N_{2} \varepsilon\right. \\
& \leq M\left(\mid\left(D_{H K}\right) \int_{t_{2}}^{t_{1}} e^{P(s)}(p(s) D v(s)-f(s)-g(s, v(s), D v(s)) \mathrm{d} s \mid\right. \\
& \quad+\mid\left(D_{H K}\right) \int_{t_{2}}^{t_{1}} e^{P(s)}(p(s) D u(s)-f(s)-g(s, u(s), D u(s)) \mathrm{d} s \mid)+N_{2} \varepsilon .
\end{aligned}
$$

Since $e^{P(t)}(p(t) v(t)+D v(t))$ and $e^{P(t)}(p(t) v(t)+D u(t))$ are $D_{H K}$-integrable on $[0, T]$, the primitives of $e^{P(t)}(p(t) v(t)+D v(t))$ and $e^{P(t)}(p(t) v(t)+D u(t))$ are continuous and so are uniformly continuous on $[0, T]$. Similarly, the primitives of $e^{P(t)}(p(t) D v(t)-f(t)-g(t, v, D v))$ and $e^{P(t)}(p(t) D u(t)-f(t)-g(t, v, D u))$ are uniformly continuous on $[0, T]$. Therefore, by inequalities (15) and (16), $G_{1}([\varphi, \psi])$ and $G_{2}([\varphi, \psi])$ are equiuniformly continuous on $[0, T]$ for all $(x, y) \in[\varphi, \psi]$. So $G([\varphi, \psi])$ is equiuniformly continuous on $[0, T]$ for all $(x, y) \in[\varphi, \psi]$.

In view of the Ascoli-Arzelàtheorem, $G([\varphi, \psi])$ is relatively compact. This result implies that $G$ satisfies the hypotheses of Lemma 3.2, whence $G$ has the minimal fixed point $x_{*}=(\underline{x}, \underline{y})$ and the maximal fixed point $x^{*}=(\bar{x}, \bar{y})$. It follows from Lemma 3.1 that $\underline{x}, \bar{x}$ are solutions of PBVP (1), and that $D \underline{x}=\underline{y}$ and $D \bar{x}=\bar{y}$.

Let $\varphi_{0}=\varphi, \psi_{0}=\psi$, and $\varphi_{n}=\bar{G} \varphi_{n-1}$,

$\psi_{n}=G \psi_{n-1}(n=1,2,3, \cdots)$, then (3.8) and (3.9) hold. If $x \in[v, u]$ with $D x \in[D v, D u]$ is a solution of (1), it follows from Lemma 3.1 that $z=(x, D x)$ is a fixed point of $G$. It follows from the extremality of $x_{*}$ and $x^{*}$ that $x_{*} \leq z \leq x^{*}$, i.e., $v \leq x \leq u$ and $D v \leq D x \leq D u$.

As a consequence of Theorem 3.1 we have 
Corollary 3.1. Given the functions $f_{1}, f_{2}$, assume that conditions $(D 0)$ and $(D 1)$ hold for the function

$$
\begin{aligned}
& g(t, x, y)=f_{1}(t, x)+f_{2}(t, y), \\
& t \in[0, T], x, y \in C([0, T]) .
\end{aligned}
$$

If $f_{1}(t, \cdot)$ is nonincreasing in $[v(t), u(t)]$ for all $t \in[0, T]$, and if $f_{2}(t, \cdot)$ is nonincreasing in $[D v(t), D u(t)]$ for all $t \in[0, T]$, then the PBVP (1.1) has the extremal solutions in $[v, u]$.

\section{REFERENCES}

[1] S. Leela, "Monotone Method for Second Order Periodic Boundry Value Problems," Nonlinear Analysis, Vol. 7, No. 4, 1983, pp. 349-355. doi:10.1016/0362-546X(83)90088-3

[2] V. Lakshmikantham, V. Sree Hari Rao and A. S. Vatsala, "Monotone Method for a System of Second Order Periodic Boundary Value Problems," Applied Mathematics Computation, Vol. 15, 1984, pp. 71-83. doi:10.1016/0096-3003(84)90054-7

[3] E. Talvila, "The Distributional Denjoy Integral," Real
Analysis, Exchang, Vol. 33, No. 1, 2008, pp. 51-82.

[4] P. Y. Lee, "Lanzhou Lecture on Henstock Integration," World Scientific, Singapore City, 1989.

[5] Š. Schwabik and G. J. Ye, "Topics in Banach Space Integration," World Scientific, Singapore City, 2005.

[6] Y. P. Lu, G. J. Ye and Y. Wang, "The Darboux Problem Involving the Distributional Henstock-Kurzweil Integral," Proceedings of the Edinburgh Mathematical Society, Vol. 55, No. 1, 2012, pp. 197-205.

[7] Y. P. Lu, G. J. Ye and W. Liu, "Existence of Solutions of the Wave Equation Involving the Distributional Henstock-Kurzweil Integral," Differential Integral Equation, Vol. 24, No. 11-12, 2011, pp. 1063-1071.

[8] Q. L. Liu and G. J. Ye, "Some Problems on the Convergence of the Distributional Denjoy Integral," Acta Mathematics Sinica, Vol. 54, No. 4, 2011, pp. 659-664.

[9] D. D. Ang, K. Schmitt and L. K. Vy, "A Multidimensional Analogue of the Denjoy-Perron-Henstock-Kurzweil Integral," Bulletin of the Belgian Mathematical Society, Vol. 4, No. 3, 1997, pp. 355-371.

[10] D. J. Guo, Y. J. Cho and J. Zhu, "Partial Ordering Methods in Nonlinear Problems," Hauppauge, Nova Science Publishers, New York, 2004. 